\title{
Pharmacogenetic Excitation of Dorsomedial Prefrontal Cortex Restores Fear Prediction Error
}

\author{
Joanna Oi-Yue Yau and Gavan P. McNally \\ School of Psychology, The University of New South Wales, Sydney 2052, New South Wales, Australia
}

Pavlovian conditioning involves encoding the predictive relationship between a conditioned stimulus (CS) and an unconditioned stimulus, so that synaptic plasticity and learning is instructed by prediction error. Here we used pharmacogenetic techniques to show a causal relation between activity of rat dorsomedial prefrontal cortex (dmPFC) neurons and fear prediction error. We expressed the excitatory hM3Dq designer receptor exclusively activated by a designer drug (DREADD) in dmPFC and isolated actions of prediction error by using an associative blocking design. Rats were trained to fear the visual CS (CSA) in stage I via pairings with footshock. Then in stage II, rats received compound presentations of visual CSA and auditory CS (CSB) with footshock. This prior fear conditioning of CSA reduced the prediction error during stage II to block fear learning to CSB. The group of rats that received AAV-hSYN-eYFP vector that was treated with clozapine- $\mathrm{N}$-oxide (CNO; $3 \mathrm{mg} / \mathrm{kg}$, i.p.) before stage II showed blocking when tested in the absence of $\mathrm{CNO}$ the next day. In contrast, the groups that received AAV-hSYN-hM3Dq and AAV-CaMKII $\alpha-\mathrm{hM} 3 \mathrm{Dq}$ that were treated with CNO before stage II training did not show blocking; learning toward CSB was restored. This restoration of prediction error and fear learning was specific to the injection of CNO because groups that received AAV-hSYN-hM3Dq and AAV-CaMKII $\alpha-\mathrm{hM} 3 \mathrm{Dq}$ that were injected with vehicle before stage II training did show blocking. These effects were not attributable to the DREADD manipulation enhancing learning or arousal, increasing fear memory strength or asymptotic levels of fear learning, or altering fear memory retrieval. Together, these results identify a causal role for dmPFC in a signature of adaptive behavior: using the past to predict future danger and learning from errors in these predictions.

Key words: blocking; DREADDs; prediction error; prefrontal cortex

\section{Introduction}

Pavlovian fear conditioning enables learning about, and adaptive responding to, sources of danger. Central to this learning is encoding the predictive relationship between a conditioned stimulus (CS) and an aversive unconditioned stimulus (US). Such encoding is instructed by prediction error (Rescorla and Wagner, 1972; Sutton, 1988). If the aversive event is expected, then there is no error in prediction and fear learning is blocked, but, if it is unexpected, then there is prediction error so that synaptic plasticity and fear learning occur (McNally et al., 2011). In this way, prediction error directs learning toward cues providing novel information about the occurrence of the outcome.

Whereas amygdala has long been implicated in fear via its obligatory role in pavlovian fear conditioning (Maren and Quirk, 2004), the medial prefrontal cortex [anterior cingulate cortex (ACC) and dorsolateral prefrontal cortex (dlPFC) in primates, and their homologues in the rodent dorsomedial PFC (dmPFC)] is considered central to the generation and signaling of prediction error (Corlett et al., 2004, 2006; Matsumoto et al., 2007; Rush-

Received Sept. 9, 2014; revised Oct. 13, 2014; accepted Nov. 5, 2014.

Author contributions: J.O.-Y.Y. and G.P.M. designed research; J.0.-Y.Y. performed research; J.O.-Y.Y. and G.P.M. analyzed data; J.0.-Y.Y. and G.P.M. wrote the paper.

This research was supported by an Australian Postgraduate Award to J.0.-Y.Y., and by Grants FT120100250 from the Australian Research Council and Grant 1003058 from the National Health and Medical Research Council to G.P.M.

Correspondence should be addressed to Dr. Gavan P. McNally, School of Psychology, The University of New South Wales, Sydney 2052, NSW, Australia. E-mail: g.mcnally@unsw.edu.au.

DOI:10.1523/JNEUROSCI.3777-14.2015

Copyright $\odot 2015$ the authors $\quad 0270-6474 / 15 / 350074-10 \$ 15.00 / 0$ worth and Behrens, 2008; Alexander and Brown, 2011; McNally et al., 2011). In humans, unexpected aversive events evoke greater fMRI BOLD responses in ACC and dlPFC compared with expected aversive events (Dunsmoor et al., 2008; Dunsmoor and Schmajuk, 2009; Eippert et al., 2012); and likewise in rodents and monkeys, unexpected aversive events evoke greater dmPFC activity than expected aversive events (Matsumoto et al., 2007; Furlong et al., 2010). Moreover, the magnitude of these evoked signals is negatively correlated with current predictions of danger (Dunsmoor et al., 2008) but is positively correlated with how much fear learning occurs on a trial (Eippert et al., 2012). Together, these findings have been interpreted to mean that medial PFC (mPFC) contributes to signaling or encoding of fear prediction errors and the consequent optimization of learning and behavior.

However, a fundamental difficulty in evaluating this prediction error hypothesis of $\mathrm{dmPFC}$ function is that existing evidence relies heavily on correlative neuroimaging and in vivo electrophysiological data. Whereas the role of $\mathrm{MPFC}$ in regulating fear responding is well established (Milad and Quirk, 2002; Quirk et al., 2006; Vidal-Gonzalez et al., 2006), little causal evidence implicates dmPFC as a component of the fear prediction error circuitry. To address this issue, we adopted a pharmacogenetic approach, using adenoviral vectors to transfect rat dmPFC neurons with the excitatory $\mathrm{hM} 3 \mathrm{Dq}$ designer receptor exclusively activated by a designer drug (DREADD; Armbruster et al., 2007; Alexander et al., 2009; Rogan and Roth, 2011). We manipulated 
the activity of these neurons via injections of clozapine- $N$-oxide $(\mathrm{CNO})$ during behavioral associative blocking procedures that selectively isolated the contribution of prediction error to fear learning. If increases in dmPFC activity signal unexpected aversive events and errors in fear predictions, then pharmacogenetic excitation of these neurons should be sufficient to restore prediction error and fear learning under conditions where these are otherwise absent.

\section{Materials and Methods}

\section{Subjects}

Subjects were 132 experimentally naive male Sprague Dawley rats (250$380 \mathrm{~g}$ ) obtained from the Animal Resources Centre (Murdoch). Rats were housed in groups of four in a climate-controlled colony room maintained on a $12 \mathrm{~h}$ light/dark cycle (lights on 7:00 A.M). Rats were food restricted to $85-90 \%$ of their free-feeding weight (for behavioral experiments) or otherwise maintained on ad libitum access to food and water. All procedures were performed in accordance with the Animal Care and Ethics Committee (University of New South Wales) and the Australian Code for the Care and Use of Animals for Scientific Purposes (eighth edition).

\section{Apparatus}

Behavioral testing was conducted in a set of 16 identical Med-Associates chambers $(24 \mathrm{~cm}$ length $\times 30 \mathrm{~cm}$ width $\times 21 \mathrm{~cm}$ height) enclosed in ventilated, sound-attenuating cabinets $(83 \mathrm{~cm}$ length $\times 59 \mathrm{~cm}$ width $\times$ $59 \mathrm{~cm}$ height). The top, rear, and hinged door of the chamber were constructed of clear Perspex, while the sidewalls were made of stainless steel. Each chamber floor contained a metal grid made of steel rods wired to a constant-current generator to deliver a footshock US. The sidewall of each box was fitted with a magazine dish $(5 \times 5 \mathrm{~cm}$ opening $)$ where grain pellets (Able Scientific Biotechnology) were delivered when a lever located $4 \mathrm{~cm}$ to the right of the magazine was pressed. The visual CS (CSA) was a $60 \mathrm{~s}$ flashing $(2 \mathrm{~Hz})$ LED light mounted to the roof of the soundattenuating cabinet, and the auditory CS (CSB) was a $60 \mathrm{~s}, 80 \mathrm{~dB}$ clicker delivered through a speaker attached to the right side wall of the chamber. The US was a $0.5 \mathrm{~s}$ footshock that varied in intensity $(0.6,0.8$, or 1 $\mathrm{mA}$ ), depending on the experimental procedures.

\section{Viral vectors}

Adeno-associated virus (AAV) vectors encoding DREADD (Armbruster et al., 2007; Rogan and Roth, 2011) were obtained from the UNC Vector Core (University of North Carolina, Chapel Hill, NC). The following vectors were used: AAV5-hSyn-enhanced yellow fluorescent protein [eYFP; $4 \times$ $10^{12}$ viral particles (vp)/ml titer]; AAV5-hSyn-HA-hM3D(Gq)-IRESmCitrine $\left(2 \times 10^{12} \mathrm{vp} / \mathrm{ml}\right)$; and AAV5-CaMKII $\alpha$-HA-hM3D(Gq)-IRESmCitrine $\left(3 \times 10^{12} \mathrm{vp} / \mathrm{ml}\right)$.

\section{Surgery}

Rats were injected intraperitoneally with a cocktail of the anesthetic ketamine $(100 \mathrm{mg} / \mathrm{ml})$ and the muscle relaxant xylazine $(0.3 \mathrm{ml} / \mathrm{kg})$. Once anesthetized, each rat was placed into the stereotaxic apparatus (model 942 , Kopf) and the rat's head was shaved to expose the skin surface. After incision, a hand drill was used to make two holes above the dorsomedial prefrontal cortex: anteroposterior +3.0 , mediolateral \pm 0.5 , dorsoventral -4.3 according to the atlas of Paxinos and Watson (2007). A 30-gauge, cone-tipped needle attached to a $5 \mu$ lglass Hamilton syringe was lowered into the dmPFC. Each hemisphere received a $0.75 \mu \mathrm{l}$ infusion at a rate of $0.25 \mu \mathrm{l} / \mathrm{min}$ as controlled by a microinfusion pump (World Precision Instruments). The needle was left in place for an additional $7 \mathrm{~min}$ to allow for diffusion. Bone wax (Coherent Scientific) was placed over the drilled holes and rats were injected intraperitoneally with $0.3 \mathrm{ml}$ of a $300 \mathrm{mg} / \mathrm{ml}$ solution of procaine penicillin (antibiotic), subcutaneously with $0.1 \mathrm{ml}$ of a $100 \mathrm{mg} / \mathrm{ml}$ solution of cefazolin sodium (antibiotic), and subcutaneously with $5 \mathrm{mg} / \mathrm{ml}$ carprofen (anti-inflammatory) immediately after surgery. Rats were allowed 7 recovery days before the start of any training, during which time they were handled and weighed daily.

Experiment 1 rats were all injected with AAV5-hSyn-HA-hM3D(Gq)IRES-mCitrine vector. Rats in experiment 2 were randomly assigned to injections of AAV5-hSyn-eYFP, AAV5-hSyn-HA-hM3D(Gq)-IRESmCitrine, or AAV5-CaMKII $\alpha$-HA-hM3D(Gq)-IRES-mCitrine injections. Rats in experiment 3 were randomly assigned to receive injections of either AAV5-hSyn-eYFP or AAV5-hSyn-HA-hM3D(Gq)-IRES-mCitrine. $\mathrm{CNO}$ was injected 3 weeks after virus injection.

Experiment 1: Effects of CNO activation of $h M 3 D q D R E A D D$ on neuronal activity in $\mathrm{dmPFC}$

Three weeks after surgery, rats were briefly removed from their home cages and injected intraperitoneally with $3 \mathrm{mg} / \mathrm{kg}$ CNO (National Institute of Mental Health Chemical Synthesis and Drug Supply Program, RTI International; 5\% DMSO and $0.9 \%$ saline solution) or a vehicle (Veh; 5\% DMSO and $0.9 \%$ saline solution). One hundred fifty minutes later, rats were deeply anesthetized with sodium pentobarbital and perfused, and brain tissue was extracted for immunohistochemistry.

\section{Experiment 2: Effects of CNO activation of dmPFC hM3Dq on associative blocking of fear learning}

In these experiments, we used conditioned suppression of lever pressing for food to assess pavlovian conditioned fear. Conditioned suppression as a measure of fear has several advantages. Conditioned suppression has a nonzero baseline because rats lever press for a pellet reward at a constant rate, and so can reveal decreases and increases in fear; there are relatively high levels of baseline activity during training and testing sessions; it is sensitive to visual and auditory CSs, despite these CSs eliciting different amounts of freezing on the same trial (Bevins and Ayres, 1991); each of the seminal behavioral preparations identifying the actions of prediction error on fear association formation was established using conditioned suppression; and, finally, the assessment of conditioned suppression is completely automated.

Baseline lever-pressing training and CS pre-exposure. All behavioral testing began with lever-pressing training to develop a baseline measure for conditioned suppression. On days 1 and 2, rats received magazine training where every lever press was rewarded with a grain pellet in addition to free pellet deliveries on a fixed interval $300 \mathrm{~s}$ schedule. Sessions ended after $60 \mathrm{~min}$ or when 100 lever presses were reached. Rats were hand shaped during day 2 if they failed to emit 100 lever presses on day 1 . On day 3, rats received a $60 \mathrm{~min}$ variable interval (VI) $30 \mathrm{~s}$ session. Subsequently, and unless otherwise noted, rats received 120 min VI $120 \mathrm{~s}$ sessions. On days 9 and 10, rats were familiarized and habituated to the CSs during 120 min VI 120 s schedule training. In each session, CSA and CSB were presented four times each in a randomized order with a randomized intertrial interval (ITI) ranging from 1200 to $1800 \mathrm{~s}$.

Stage I. Stage I occurred for $3 \mathrm{~d}$. Blocking groups received four pairings of CSA and US $(0.8 \mathrm{~mA})$ on a randomized ITI ranging from 1200 to 1800 s during a 120 min session. Rats in control groups did not receive stage I training but instead received $120 \mathrm{~min}$ of VI $120 \mathrm{~s}$ schedule training.

Stage II. All rats underwent stage II training on days 14 and 15 during 70 min of VI $120 \mathrm{~s}$ schedule training. During this stage, CSA and CSB were presented in compound for $1 \mathrm{~min}$, coterminating with the US (0.8 $\mathrm{mA})$. CSAB-shock pairings were presented four times with a randomized ITI ranging from 490 to $1200 \mathrm{~s}$. Rats were injected with CNO $(3 \mathrm{mg} / \mathrm{kg}$, i.p.) or vehicle $30 \mathrm{~min}$ before each session.

Test. All rats were tested on day 16. The test was $70 \mathrm{~min}$ long, and CSB was presented four times on an ITI of $900 \mathrm{~s}$.

\section{Experiment 3: Effects of CNO activation of dmPFC hM3Dq on} acquisition and expression of fear conditioning

Baseline lever-pressing training and CS pre-exposure. The procedure for baseline lever pressing and CS pre-exposure was the same as in experiment 2.

Stage I. This stage examined the effects of $\mathrm{CNO}$ on the acquisition of pavlovian fear learning. Rats underwent fear conditioning to the visual CS. Groups received four pairings of a visual CS with either a 0.6 or $1 \mathrm{~mA}$ US on a randomized ITI ranging from 1200 to $1800 \mathrm{~s}$ during a $120 \mathrm{~min}$ session. All rats received intraperitoneal injections of $3 \mathrm{mg} / \mathrm{kg}$ CNO 30 min before each session. On the subsequent 3 days, rats were tested drug free. In each 70 min test session, four CS non-reinforced trials were presented with a randomized ITI ranging from 490 to 1200 s. Rats were not trained on day 17 
Stage II. This stage examined the effects of CNO activation of dmPFC $\mathrm{hM} 3 \mathrm{Dq}$ on the expression of fear conditioning. The rats trained in stage I with the $0.6 \mathrm{~mA}$ US were trained again in stage II. This training, which occurred on days $18-20$, was identical to that in stage I, except that an auditory CS coterminated with a $0.6 \mathrm{~mA}$ US and there were no injections before training. On days $21-23$, rats were tested in 70 min test sessions involving four non-reinforced CS presentations with a randomized ITI ranging from 490 to $1200 \mathrm{~s}$. Rats were injected with $\mathrm{CNO}(3 \mathrm{mg} / \mathrm{kg}$, i.p) 30 min before each test day.

\section{Immunohistochemistry}

Experiment 1. Rats were deeply anesthetized with sodium pentobarbital and transcardially perfused with saline containing $1 \%$ sodium nitrate and heparin $(5000 \mathrm{IU} / \mathrm{ml})$ and paraformaldehyde (4\%), $\mathrm{pH} \mathrm{7.4,} 2.5 \mathrm{~h}$ after an intraperitoneal injection of either $3 \mathrm{mg} / \mathrm{kg} \mathrm{CNO}$ or vehicle. Brains were extracted, postfixed ( $1 \mathrm{~h}$ ), and cryoprotected in $20 \%$ sucrose (24-48 h). Brains were frozen, and $40 \mu \mathrm{m}$ PFC coronal sections were sliced and collected on a cryostat (CM 1950, Leica) and stored in a $0.1 \mathrm{M}$ $\mathrm{PB}$ saline solution containing $0.1 \%$ sodium azide at $4^{\circ} \mathrm{C}$. Two-color peroxidase immunohistochemistry was used to reveal the activity marker c-Fos and green fluorescent protein (GFP) immunoreactivity (IR) to detect cells expressing mCitrine. Free-floating brain tissue was washed in $\mathrm{PB}, \mathrm{pH} 7.4$, and rinsed in alcohol (50\%), alcohol containing hydrogen peroxide (3\%), and normal horse serum (NHS; $5 \%$ ) in $0.1 \mathrm{M} \mathrm{PB,} \mathrm{pH} \mathrm{7.4,}$ for $30 \mathrm{~min}$ each. Sections were then incubated in rabbit anti-GFP (1: 2000; Life Technologies) and goat anti-c-Fos [1:2000; c-Fos (4), sc52 Santa Cruz Biotechnology] for $48 \mathrm{~h}$ at $4^{\circ} \mathrm{C}$. The primary antibodies were diluted in PBT-X, comprising $0.1 \mathrm{M} \mathrm{PB}, \mathrm{pH} 7.4$, containing $0.1 \%$ sodium azide, $2 \%$ NHS, and $0.02 \%$ Triton X-100. After washing off unbound primary antibody, sections were incubated overnight (at room temperature) in biotinylated donkey anti-sheep IgG (1:2000; Jackson ImmunoResearch) diluted in 2\% NHS PBT-X. After washing off unbound secondary antibody, sections were incubated for $2 \mathrm{~h}$ (at room temperature) in $\mathrm{ABC}$ reagent (Vector Elite Kit $6 \mu \mathrm{l} / \mathrm{ml}$ avidin and $6 \mu \mathrm{l} / \mathrm{ml}$ biotin; Vector Laboratories). Black c-Fos-IR cells were revealed using an intensified diaminobenzidine $(\mathrm{DAB})$ reaction, with peroxide being generated by glucose oxidase. In this $\mathrm{DAB}$ reaction, sections were washed in $\mathrm{PB}, \mathrm{pH}$ 7.4, followed by $0.1 \mathrm{~m}$ acetate buffer, $\mathrm{pH}$ 6.0, and then incubated for 15 $\mathrm{min}$ in a $0.1 \mathrm{M}$ acetate buffer, $\mathrm{pH} 6.0$, solution containing $2 \%$ nickel sulfate, $0.025 \% \mathrm{DAB}, 0.04 \%$ ammonium chloride, and $0.02 \% \mathrm{D}$-glucose. The peroxidase reaction was started by adding $0.1 \mu \mathrm{l} / \mathrm{ml}$ glucose oxidase and stopped using acetate buffer, $\mathrm{pH}$ 6.0. Brain sections were then washed in $\mathrm{PB}, \mathrm{pH} 7.4$, and processed again, in a similar manner, using biotinylated donkey anti-rabbit IgG (1:5000; Jackson ImmunoResearch) but without nickel-intensification to localize brown GFP-IR. Sections were then washed in PB, pH 7.4, and mounted onto gelatin-treated slides, dehydrated, cleared with histolene, and coverslipped with Entellan.

c-Fos-IR and double c-Fos/GFP-IR were counted using a transmitted light microscope at $20 \times$ magnification. The dmPFC was delineated according to Paxinos and Watson (2007), and, across three sections, neurons positive for c-Fos-IR and double c-Fos/GFP-IR were counted in each hemisphere.

Experiments 2 and 3. The localization of hM3Dq or eYFP expression was verified using procedures identical to those described above. However, rats did not receive injections before perfusions, and tissue was only processed for brown-colored GFP-IR to detect cells expressing eYFP or mCitrine using DAB reaction without nickel intensification. eYFP and hM3Dq expression sites were determined under a microscope and plotted onto Illustrator CS6 (Adobe Systems) templates using boundaries defined by Paxinos and Watson (2007). Rats with unilateral expression or expression outside the boundaries of the anterior cingulate cortex and prelimbic cortex were excluded from data analysis.

Data analysis. The lever pressing of each rat was scored using a suppression ratio (SR) calculated as SR $=a /(a+b)$ (Annau and Kamin, 1961), where $a$ is the number of lever presses during the CS and $b$ is the number of lever presses made the minute before the CS (the pre-CS period). An SR of 0.5 indicates no suppression (equal numbers of lever presses during the pre-CS and CS period), hence no fear, and an SR of 0 indicates complete suppression, hence asymptotic fear. Although lever pressing remained relatively stable, there were some trials (11 in total across the three experiments) where an animal did not lever press during the pre-CS period of the first CS presentation despite evidence of a steady lever-pressing baseline otherwise in the session. In these instances, typically due to the rat eating a recently dispensed food pellet, the pre-CS was taken at the eighth minute after session initiation. These behavioral data as well as the total counts of c-Fos-IR and c-Fos/GFP-IR neurons in $\mathrm{dmPFC}$ were analyzed by means of a planned contrasting testing procedure controlling the decision-wise error rate $(\alpha)$ at the 0.05 level for each contrast tested (Harris, 2004).

\section{Results}

\section{Experiment 1: effects of CNO activation of hM3Dq DREADD on neuronal activity in $\mathrm{dmPFC}$}

We used AAV to transfect the hM3Dq excitatory DREADD (Alexander et al., 2009; Rogan and Roth, 2011) into rat dmPFC neurons and then stimulated these neurons with $\mathrm{CNO}(3 \mathrm{mg} / \mathrm{kg}$, i.p.). To confirm that stimulation of hM3Dq increased activity in dmPFC, we first injected AAV-hSyn-HA-hM3D(Gq)-IRESmCitrine (hSyn-hM3Dq) rats with CNO $(n=4)$ or vehicle $(n=$ 5 ) and assessed expression of the Fos protein as a marker of neural activity induced by CNO (Garner et al., 2012; Ferguson et al., 2013; Zhan et al., 2013) in dmPFC neurons. An advantage of this immunohistochemical approach is that it enables examination of the impact of CNO injections on both hM3Dq-expressing and hM3Dq-nonexpressing cells. Pharmacogenetic activation was successful because, consistent with previous reports (Garner et al., 2012; Zhan et al., 2013), labeling for Fos was detected in both dmPFC hM3Dq-expressing and hM3Dq-nonexpressing cells (Fig. 1A-C). Compared with the injection of vehicle, we detected significantly more dmPFC Fos-positive nuclei $\left(F_{(1,7)}=15.3, p<\right.$ $0.05)$ and significantly more dual-labeled Fos/GFP-IR dmPFC cells after the injection of $\mathrm{CNO}\left(F_{(1,7)}=15.7, p<0.05\right.$; Fig. $\left.1 D\right)$. Indeed, relative to vehicle, $\mathrm{CNO}$ injection approximately doubled the number of c-Fos-IR and tripled the number of c-Fos-IR/ GFP-IR-expressing cells in dmPFC.

\section{Experiment 2: effects of CNO activation of dmPFC hM3Dq on associative blocking of fear learning}

To examine the causal role of dmPFC neurons in fear prediction errors, we injected rat dmPFC with either AAV-hSyn-eYFP $(n=$ 31) or AAV-hSyn-HA-hM3D(Gq)-IRES-mCitrine vector $(n=$ 32), to express the excitatory DREADD hM3Dq nonselectively in neurons, or AAV-CaMKII $\alpha-\mathrm{HA}-\mathrm{hM} 3 \mathrm{D}(\mathrm{Gq})-\mathrm{IRES}-\mathrm{mCitrine}$ $(n=31)$, to express the excitatory hM3Dq DREADD in glutamatergic neurons (Fig. $2 A, B$ ). Two weeks later, we commenced behavioral training (Fig. 2C). Associative blocking is the cardinal behavioral preparation isolating the actions of prediction error on pavlovian fear learning (Kamin, 1968; Rescorla and Wagner, 1972; McNally and Westbrook, 2006). In this paradigm, rats in the blocking group are trained to fear a CSA via pairings with shock in stage I. In stage II, CSA is presented in compound with a second CS, CSB, and paired with shock. Rats are later tested for their fear responses to CSB. Relative to a control group that does not receive stage I training, the blocking group shows significantly less fear to CSB. Blocking occurs because there is no prediction error during stage II for the blocking group: the prior conditioning of CSA ensures that the shock US is predicted during stage II, and this expected footshock is less effective in supporting learning than the unexpected footshock in the control group. Hence, the design of this experiment was a 3 (genotype: eYFP, hSyn-hM3Dq, CaMKII $\alpha$-hM3Dq) $\times 2$ (group: blocking versus control) $\times 2$ (injection: $\mathrm{CNO}$ versus vehicle) factorial. 

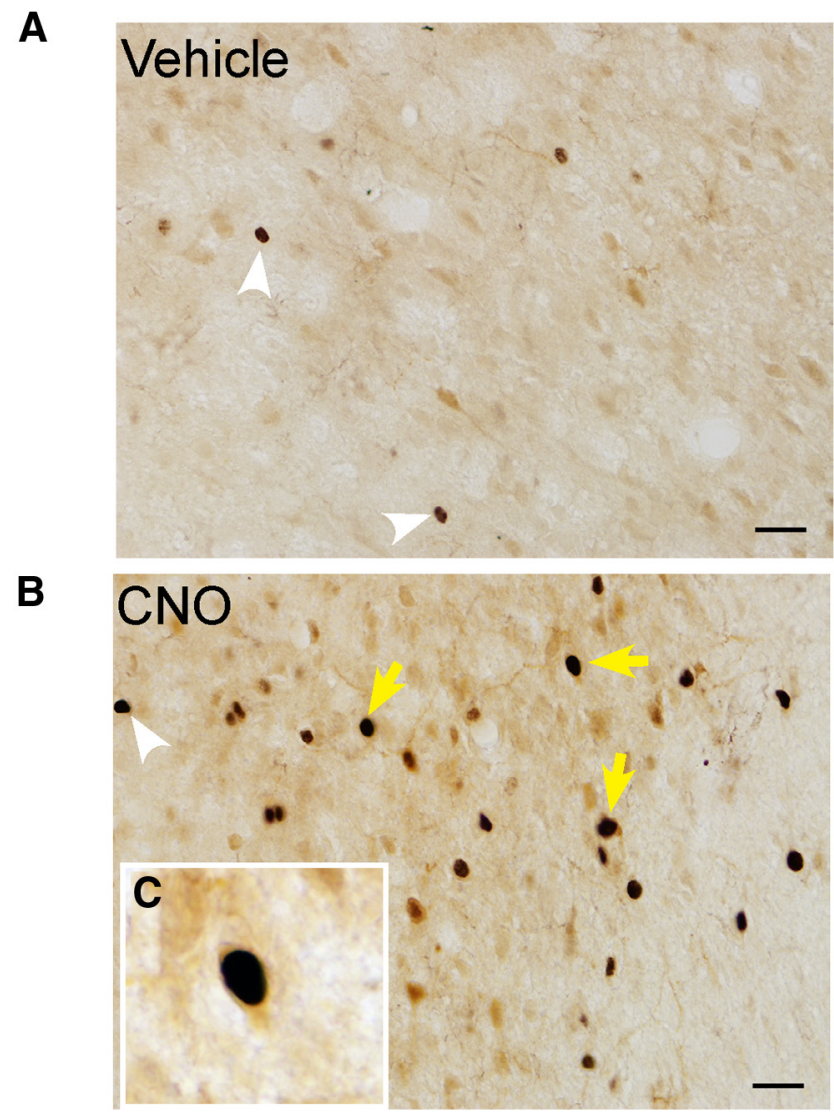

D
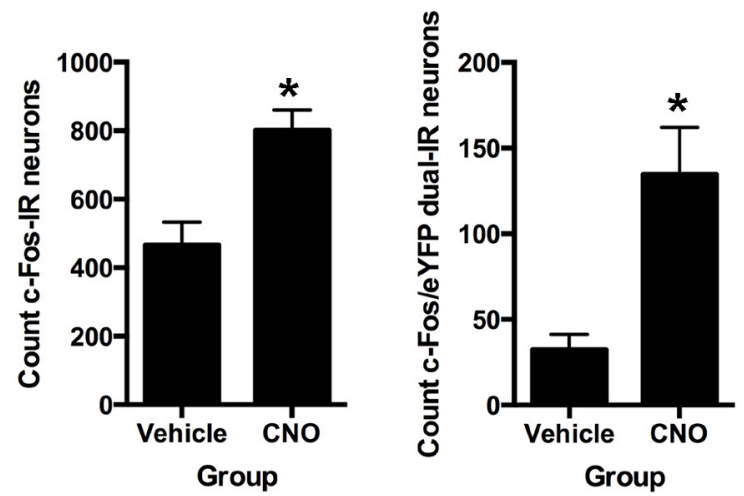

Figure 1. A, Photomicrographs showing representative c-Fos-IR for a vehicle-injected animal. $\boldsymbol{B}$, Photomicrographs showing representative C-Fos-IR for an animal injected with CNO (3 $\mathrm{mg} / \mathrm{kg}$, i.p.). C, Example dual-labeled c-Fos-IR/GFP-IR neurons in $\mathrm{dmPFC}$, D, Counts of total c-Fos-IR and total c-Fos-IR/GFP-IR dual-labeled neurons. White arrowheads show singlelabeled c-Fos-IR, and yellow arrows show dual-labeled c-Fos-IR/GFP-IR neurons. Scale bar, 25 $\mu \mathrm{m} .{ }^{*} p<0.05$ versus vehicle group. Group sizes: hSyn-hM3Dq-CN0, $n=4$; hSyn-hM3Dqvehicle, $n=5$

\section{Histology}

Figure $2 A$ shows representative dmPFC GFP-IR for each group. Figure $2 B$ shows extent of GFP-IR expression across all rats included in the experiment with each rat represented at $10 \%$ opacity. Only rats with bilateral GFP-IR in dmPFC were included in the analyses. Sixteen animals were excluded because of misplaced AAV application or lack of bilateral GFP-IR. The final group sizes after histology were as follows: CNO-block-eYFP, $n=9$; CNO-block-hSyn-hM3Dq, $n=5$; CNO-block-CaMKII $\alpha$-hM3Dq, $n=5$; CNO-control-eYFP, $n=7$; CNO-control-hSyn-hM3Dq, $n=8$; CNO-controlCaMKII $\alpha$-hM3Dq, $n=6$; Veh-block-eYFP, $n=5$; Veh-block-
hSyn-hM3Dq, $n=7$; Veh-block-CaMKII $\alpha$-hM3Dq, $n=6$; Veh-control-eYFP, $n=6$; Veh-control-hSyn-hM3Dq, $n=8$; and Veh-control-CaMKII $\alpha$-hM3Dq, $n=6$.

\section{Behavior}

To confirm that AAV-mediated hM3Dq expression did not alter lever-pressing behavior, we analyzed the last day of VI 120s training before fear conditioning. There was no difference between groups in the number of lever presses during this session (eYFP: mean $=2163$, SEM $=196$; hSyn-hM3Dq: mean $=2770$, SEM $=$ 376; CaMKII $\alpha$-hM3Dq: mean $=2076$, SEM $=251 ; F_{(1,75)}$ values $<1, p$ values $>0.05$ ). During stage I fear conditioning (Fig. $2 D, E)$, the blocking groups learned to fear the visual CSA across training as shown by a linear increase of conditioned fear across the 3 days (main effect of day: $F_{(1,31)}=411.01, p<0.05$ ). There was no main effect of genotype (hSyn-eYFP versus hSyn-hM3Dq and CaMKII $\alpha$-hM3Dq; $\left.F_{(1,31)}<1, p>0.05\right)$ and no day $\times$ genotype interaction $\left(F_{(1,31)}<1, p>0.05\right)$.

We injected rats with $\mathrm{CNO}$ or vehicle before each day of stage II training. The blocking groups continued to express fear to the compound of the visual CSA and auditory CSB during stage II, regardless of whether they had been injected with $\mathrm{CNO}$ or vehicle. The control groups learned to fear the compound of the visual CSA and auditory CSB during stage II also regardless of whether they had been injected with CNO or vehicle (Fig. 2D,E). Indeed, during stage II, there was a main effect of group (blocking versus control; $\left.F_{(1,66)}=299.35, p<0.05\right)$, a main effect of day $\left(F_{(1,66)}=310.67, p<0.05\right)$, no main effect of genotype (eYFP, hSyn-hM3Dq, CaMKII $\alpha$-hM3Dq; $\left.F_{(1,66)}<1, p>0.05\right)$, and no main effect of injection (CNO versus vehicle; $F_{(1,66)}<1, p>$ $0.05)$. There was significantly more fear learning in the control than the blocking groups across stage II, as shown by the group (blocking versus control) $\times$ day interaction $\left(F_{(1,66)}=266.8, p<\right.$ $0.05)$, but there were no other differences between groups. So, pharmacogenetic excitation of dmPFC neurons did not alter fear memory retrieval or fear expression during the stage II conditioning phase for blocking groups, nor did it alter fear acquisition for the control group.

Nonetheless, pharmacogenetic excitation of dmPFC neurons did restore learning during stage II because associative blocking on the test was absent in CNO-block-hSyn-hM3Dq and CNOblock-CaMKII $\alpha$-hM3Dq groups (Fig. 2D,E). On testing, rats made a total average of 15.84 lever presses per minute $(\mathrm{SEM}=$ 1.26 ), with no differences between groups (largest $F$ value $=2.8$, $p$ value $>0.05)$. There was evidence for the associative blocking of fear learning because control groups were significantly more afraid than block groups $\left(F_{(1,66)}=66.21, p<0.05\right)$. CNO injections or DREADD expression in dmPFC alone did not exert any effect on learning as there were no overall differences between groups treated with CNO versus vehicle $\left(F_{(1,66)}=2.63, p>0.05\right)$ and no overall effect of genotype $\left(F_{(1,66)}=3.29, p>0.05\right)$. There was a group (block versus control) $\times$ drug $(\mathrm{CNO}$ versus vehicle $) \times$ genotype (eYFP versus hM3Dq) three-way interaction $\left(F_{(1,66)}=\right.$ $9.38, p<0.05)$, showing that injection of $\mathrm{CNO}$ but not vehicle during stage II prevented associative blocking in hSyn-hM3Dq and CaMKII $\alpha$-hM3Dq groups.

Further analyses showed that for groups injected with $\mathrm{CNO}$ before stage II training, blocking was prevented in the blockhSyn-hM3Dq and block-CaMKII $\alpha$-hM3Dq groups compared with the block-eYFP group $\left(F_{(1,66)}=26.59, p<0.05\right)$, and that the block-hSyn-hM3Dq and block-CaMKII $\alpha$-hM3Dq groups acquired similar levels of fear to the auditory $\operatorname{CSB}\left(F_{(1,66)}=3.11\right.$, $p>0.05)$. Despite preventing blocking, CNO had no effect on 
a
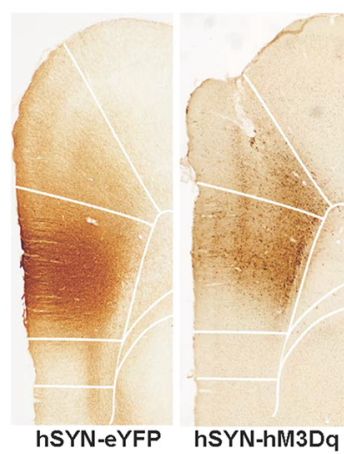

nssmatuon

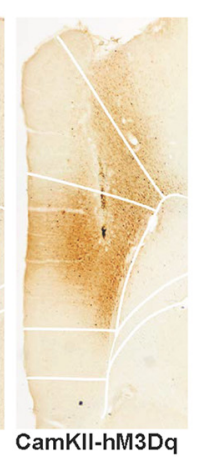

b

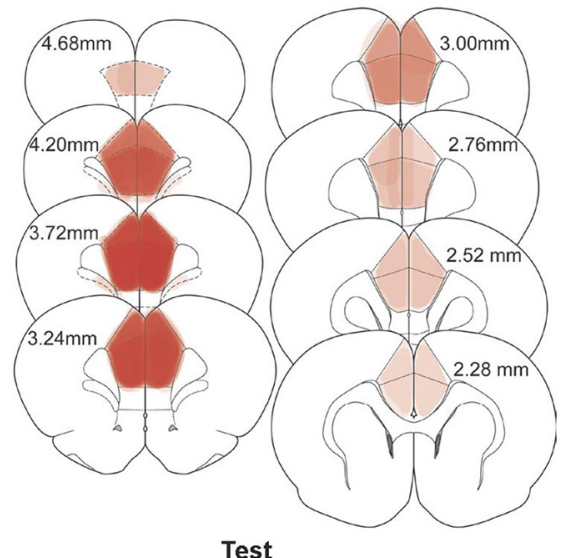

C
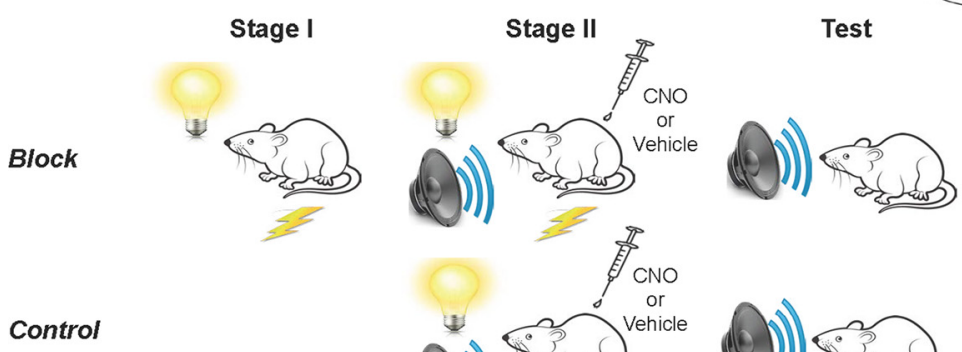
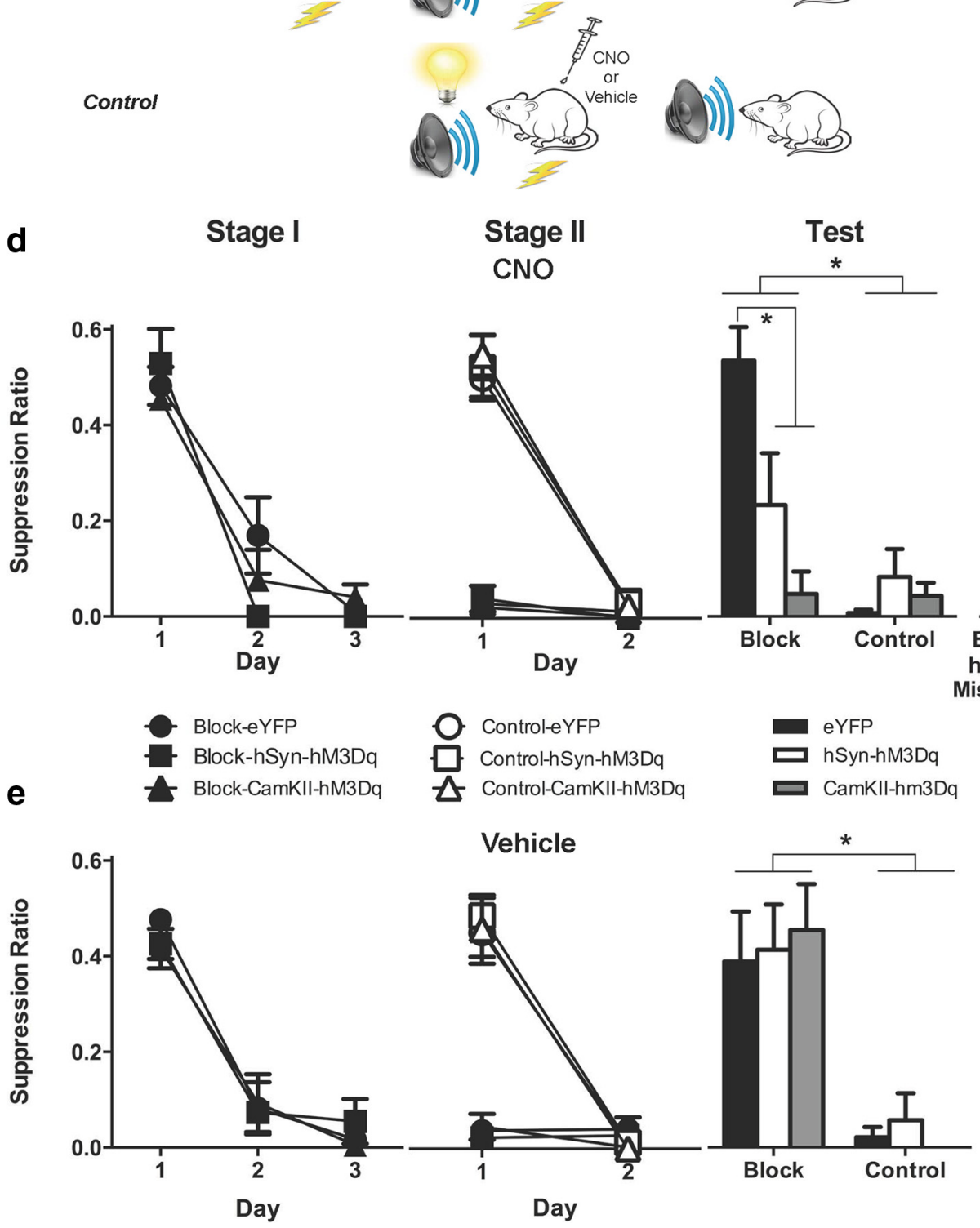

Figure 2. $\boldsymbol{a}$, Photomicrographs showing representative GFP-IR in dmPFC from hSyn-eYFP, hSyn-hM3Dq, and CaMKIll $\alpha$-hM3Dq groups. $\boldsymbol{b}$, Extent of dmPFC GFP-IR for the experiment with each rat represented at 10\% opacity. $\boldsymbol{c}$, Behavioral procedure. Blocking groups received stage I pairings of a visual CS with footshock. In stage II, both block and control groups received pairings of a auditory-visual compound CS with footshock. Rats received injections of CNO (3 mg/kg, i.p) or vehicle before stage ll training. Rats were tested for fear to the auditory CS.d, Mean \pm SEM suppression ratios during the first CS presentation each day in stages I and II, and the test for groups injected with CNO before stage II. $\boldsymbol{e}$, Mean \pm SEM suppression ratios during (Figure legend continues.) 
fear learning in the control groups $\left(F_{(1,66)}<1, p>0.05\right)$. In contrast to the $\mathrm{CNO}$ groups, injection of vehicle before stage II had no effect on blocking in the blocking groups $\left(F_{(1,66)}<1, p>\right.$ $0.05)$ or fear learning in the control groups $\left(F_{(1,66)}<1, p>0.05\right)$.

Some animals in groups CNO-block-hSyn-hM3Dq and CNO-block-CaMKII $\alpha$-hM3Dq were not included in these analyses due to misplaced or unilateral applications of AAV-hM3Dq. Hence, any pharmacogenetic excitation in these animals either was not limited to dmPFC or was only unilateral. These animals were combined to form a control group, CNO-block-hM3Dqmisplaced ( $n=6$; Fig. $2 D$ ) to determine the specificity of the effects of pharmacogenetic dmPFC excitation on fear learning. Analyses showed that the CNO-block-hM3Dq-misplaced group did not differ in fear from the block-eYFP group $\left(F_{(1,71)}=1.00\right.$, $p>0.05)$, but was significantly less afraid of the CS than the block-hSyn-hM3Dq and block-CaMKII $\alpha$-hM3Dq groups $\left(F_{(1,71)}=11.61, p<0.05\right)$.

\section{Experiment 3: effects of CNO activation of dmPFC hM3Dq on acquisition and expression of fear conditioning}

Did pharmacogenetic excitation of dmPFC neurons prevent associative blocking by restoring prediction error during stage II or did it do so by increasing shock reactivity, fear, asymptotic levels of fear learning, or fear memory consolidation? To distinguish between these possibilities, we first trained the hSyn-eYFP group $(n=15)$ and hSyn-hM3Dq group $(n=14)$ to fear a visual CS. This CS was paired with either a weak $(0.6 \mathrm{~mA})$ or a strong $(1 \mathrm{~mA})$ footshock. We injected rats with $\mathrm{CNO}$ before each day of training (Fig. 3B). Then, we tested rats for their fear responses to the visual CS across $3 \mathrm{~d}$ with $4 \mathrm{CS}$ presentations each day in the absence of CNO. Next, we determined whether pharmacogenetic excitation of $\mathrm{dmPFC}$ changed fear memory retrieval or the expression of fear. To do this, we retrained the weak footshock groups (hSyneYFP-0.6 mA and hSyn-hM3Dq-0.6 mA) via pairings of an auditory CS with a weak footshock (0.6 mA; Fig. $3 C)$. We then tested rats for their fear responses to the auditory CS across $3 \mathrm{~d}$ with four CS presentations each day. Rats received an injection of $\mathrm{CNO}$ before each test day.

\section{Histology}

Figure $3 A$ shows the extent of GFP-IR expression across all rats in the experiment with each rat represented at $10 \%$ opacity. Five animals were excluded because of misplaced AAV application or lack of GFP-IR. The final group sizes after histology were as follows: hM3Dq-0.6 mA, $n=5$; eYFP- $0.6 \mathrm{~mA}, n=5$; hM3Dq- $1 \mathrm{~mA}$, $n=6$; and eYFP- $1 \mathrm{~mA}, n=8$.

\section{Behavior}

The mean \pm SEM suppression ratios during conditioning with the visual CS and test are shown in Figure 3D. As expected, conditioned fear increased across training and decreased across tests. Overall, there was significantly more fear in the groups trained

\footnotetext{
$\leftarrow$

(Figure legend continued.) the first CS presentation each day during stages I and II, and the test for groups injected with vehicle before stage II. Although there was overall evidence for blocking in eYFP groups, this blocking was absent in block-hSyn-hM3Dq and block-CaMKII $\alpha$-hM3Dq groups There was no effect of vehicle on blocking. ${ }^{*} p<0.05$, \#p $<0.05$ compared with block-hSyn-hM3Dq and block-CaMKII $\alpha$-hM3Dq groups. Group sizes: CNO-block-eYFP, $n=9$; CNO-block-hSyn-hM3Dq, $n=5$; CNO-block-CaMKIl $\alpha$-hM3Dq, $n=5$; CNO-control-eYFP, $n=$ 7; CNO-control-hSyn-hM3Dq, $n=8$; CNO-control-CaMKIII $\alpha$-hM3Dq, $n=6$; Veh-block-eYFP, $n=5$; Veh-block-hSyn-hM3Dq, $n=7$; Veh-block-CaMKII $\alpha$-hM3Dq, $n=6$; Veh-control-eYFP, $n=6$; Veh-control-hSyn-hM3Dq, $n=8$; Veh-control-CaMKIl $\alpha$-hM3Dq, $n=6$; and CNOblock-hM3Dq-Misplaced, $n=6$.
}

with the $1 \mathrm{~mA}$ footshock compared with the $0.6 \mathrm{~mA}$ footshock $\left(F_{(1,20)}=22.97, p<0.05\right)$. This shows that our measure was sensitive to different strengths of fear learning and memory. Nonetheless, there was no overall difference between eYFP and hM3Dq groups $\left(F_{(1,20)}<1, p>0.05\right)$. Fear increased across conditioning $\left(F_{(1,20)}=238.82, p<0.05\right)$ and decreased across tests $\left(F_{(1,20)}=81.59, p<0.05\right)$, but eYFP and hM3Dq groups did not differ in these changes in fear $\left(F_{(1,20)}\right.$ values $<1, p$ values $>0.05)$. Thus, pharmacogenetic excitation of dmPFC neurons during conditioning did not enhance fear learning or memory.

The mean \pm SEM suppression ratios during conditioning with the auditory CS and test are shown in Figure 3E. Both hSyneYFP-0.6 mA and hSyn-hM3Dq-0.6 mA groups learned to fear the auditory CS, and this fear was reduced across tests. There was no overall difference between eYFP and hM3Dq $\left(F_{(1,8)}<1, p>\right.$ $0.05)$. Fear increased across training $\left(F_{(1,8)}=292.31, p<0.05\right)$ and decreased across tests $\left(F_{(1,8)}=36.07, p<0.05\right)$, but eYFP and hM3Dq groups did not differ in these changes in fear during training $\left(F_{(1,8)}<1, p>0.05\right)$ or testing $\left(F_{(1,8)}<1, p>0.05\right)$. Thus, pharmacogenetic excitation of dmPFC neurons had no effect on either the expression of fear or its decline.

\section{Discussion}

We used a pharmacogenetic approach to study the role of dmPFC in fear prediction errors. First, we showed that injection of $\mathrm{CNO}$ into animals expressing hM3Dq in $\mathrm{dmPFC}$ induced expression of the c-Fos protein, confirming that the pharmacogenetic manipulation was successful in increasing activity in dmPFC. Next, we examined the effects of pharmacogenetic activation of dmPFC on fear prediction errors using a blocking design. We tested the possibility that increases in dmPFC activity signal unexpected aversive events and errors in fear predictions, and hence we asked whether pharmacogenetic excitation of these neurons would restore prediction error and fear learning under conditions where these were otherwise absent. There was evidence that this was the case. CNO injection into animals expressing the hM3Dq in $\mathrm{dmPFC}$ prevented the associative blocking of fear learning. This prevention of blocking depended upon the combination of $\mathrm{dmPFC}$ hM3Dq expression and injection of CNO, because associative blocking was intact in hM3Dq animals injected with vehicle and in hM3Dq animals with misplaced hM3Dq expression. Critically, during this experiment, injection of CNO had no effect in control groups not subjected to the associative blocking manipulation. The absence of any significant difference in the magnitude of fear learning restoration between the CNO-blockhSyn-hM3Dq and CNO-block-CaMKII $\alpha$-hM3Dq groups shows that pharmacogenetic excitation of dmPFC glutamatergic neurons is sufficient to prevent associative blocking of fear conditioning.

The blocking paradigm isolates the contribution of prediction error to association formation (Rescorla and Wagner, 1972; Dickinson, 1980; Mackintosh, 1983). The effects of pharmacogenetic excitation here are consistent with a restoration of fear prediction error. However, this prevention of associative blocking could have reflected another action on fear learning or memory, so we examined the effects of hM3Dq activation on the acquisition and expression of fear learning. We found that hM3Dq activation did not increase or augment the acquisition of either weak or strong fear conditioning, under conditions where our measure was otherwise sensitive to these variations in the strength of fear learning. We also found that hM3Dq activation did not alter the retrieval and expression of weak fear conditioning. So, the restoration of fear learning by injection of CNO in the 
a

b

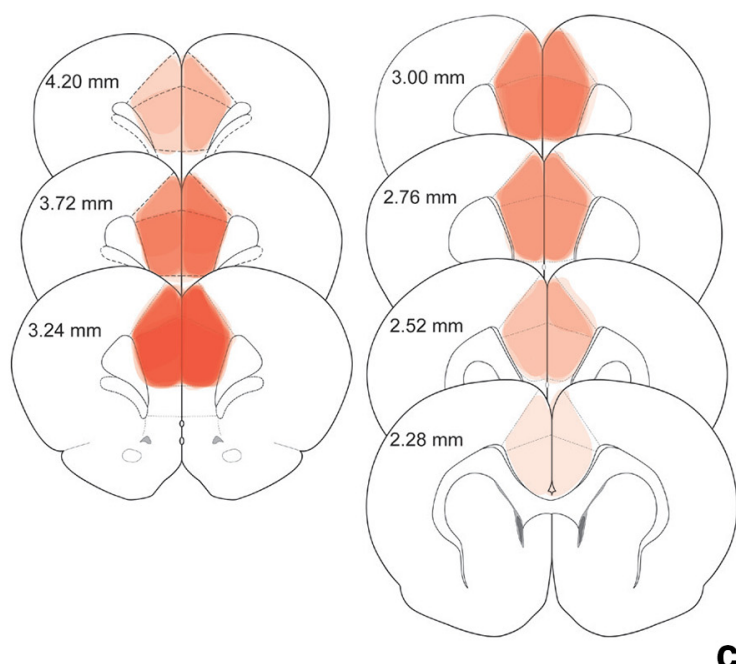

c
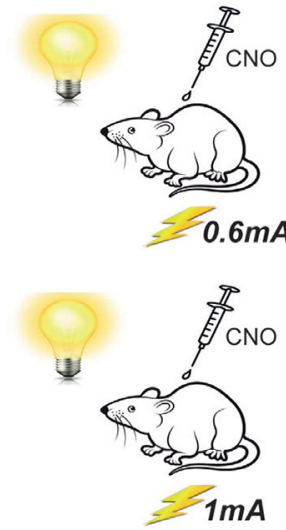

d

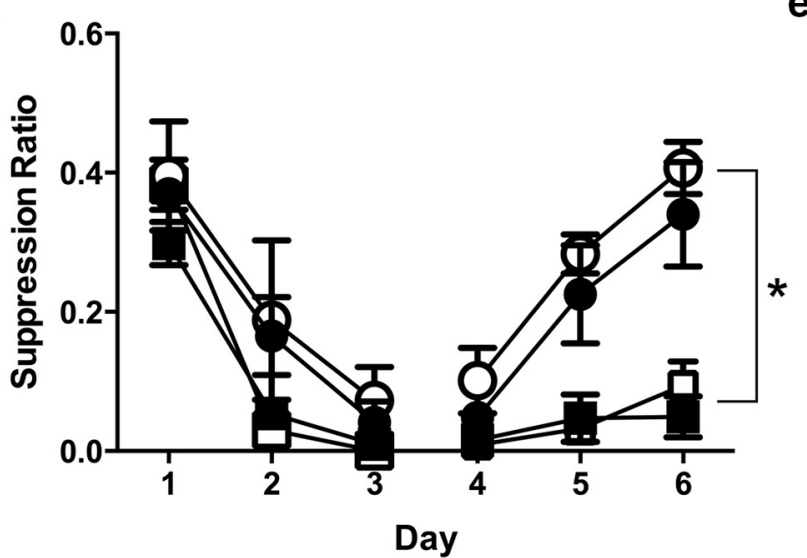

y

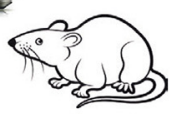

eYFP $0.6 \mathrm{~mA}$
hM3Dq $0.6 \mathrm{~mA}$
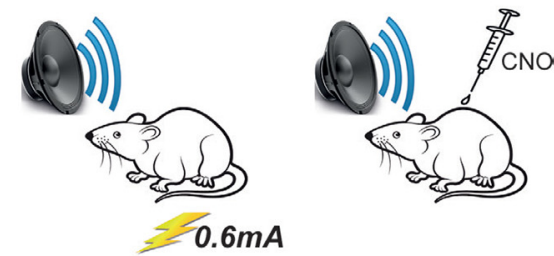

$0.6 \mathrm{~mA}$

4

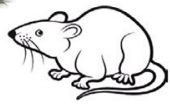

e
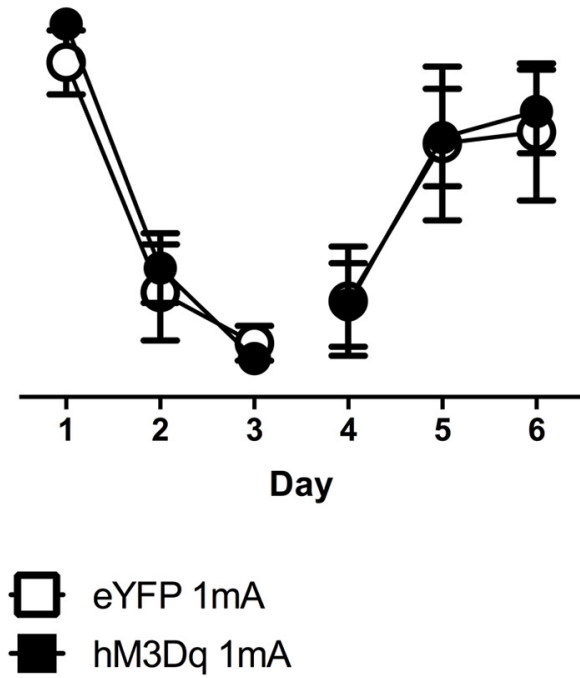

Figure 3. $\boldsymbol{a}$, Extent of dmPFC GFP-IR for the experiment, with each rat represented at $10 \%$ opacity. $\boldsymbol{b}$, Behavioral procedure. Rats received injections of $3 \mathrm{mg} / \mathrm{kg}$ CN0 then received pairings of a visual CS with a 0.6 or $1 \mathrm{~mA}$ footshock in stage I. c, In stage II, rats in the $0.6 \mathrm{~mA}$ groups were retrained with pairings of an auditory $\mathrm{CS}$ with a $0.6 \mathrm{~mA}$ footshock and then tested for fear to the auditory CS. Rats received injections of $3 \mathrm{mg} / \mathrm{kg}$ CNO before tests. $\boldsymbol{d}$, Mean \pm SEM suppression ratios during stage I. Although $1 \mathrm{~mA} \mathrm{groups} \mathrm{were} \mathrm{more} \mathrm{afraid} \mathrm{than} 0.6 \mathrm{~mA}$ groups, there was no difference between $\mathrm{hM} 3 \mathrm{Dq}$ and eYFP groups during conditioning or testing. $\boldsymbol{e}$, Mean \pm SEM suppression ratios during stage II. There was no difference between hM3Dq and eYFP groups during conditioning or testing. ${ }^{*} p<0.05$. Group sizes: hM3Dq-0.6 mA, $n=5 ;$ eYFP- $0.6 \mathrm{~mA}, n=5 ; \mathrm{hM}$ Dq-1 mA, $n=6$; eYFP- $1 \mathrm{~mA}, n=8$.

hM3Dq groups is not obviously attributable to an enhancement of fear learning, an augmentation of fear memory consolidation, an increase in asymptotic levels of learning, an increase in the functional intensity of the aversive footshock, or a change in fear memory retrieval. Based on this behavioral selectivity, we interpret these findings to mean that pharmacogenetic excitation of dmPFC neurons was sufficient to restore fear prediction error and fear learning. 


\section{Methodological considerations}

A primary consideration was whether AAV-mediated expression of the hM3Dq DREADD perturbed or otherwise disrupted dmPFC function and behavior (Armbruster et al., 2007; Alexander et al., 2009). There was no evidence for this, at least in the behaviors we assessed. Across experiments, hM3Dq groups were largely indistinguishable from eYFP controls. hM3Dq and eYFP animals acquired the baseline lever-pressing task at the same rate and to the same level; they also acquired, retained, and expressed fear at the same levels as the eYFP groups. More importantly, there were no differences at any point among the vehicle-injected eYFP, hSyn-hM3Dq, and CaMKII $\alpha$-hM3Dq groups. So, at least on measures that are directly relevant to fear learning and for interpretation of the behavioral effects reported here, in the absence of $\mathrm{CNO}, \mathrm{hM} 3 \mathrm{Dq}$ animals were indistinguishable from controls.

A second consideration was whether injection of $3 \mathrm{mg} / \mathrm{kg}$ $\mathrm{CNO}$ had off-target or nonspecific behavioral effects that could confound interpretation (Rogan and Roth, 2011). Again, there was little evidence here to support this. The CNO-injected $\mathrm{hM} 3 \mathrm{Dq}$ misplaced group displayed the same profile of fear learning as the CNO-injected eYFP control group, and $\mathrm{CNO}$ injection had no effect on fear learning or responding when hM3Dq groups did not show associative blocking. Although it remains possible that $\mathrm{CNO}$ produced nonspecific behavioral or off-target effects, there was no evidence here that any such effects confound interpretation of our primary behavioral data. Indeed, other than restoring prediction error and fear learning, injection of $\mathrm{CNO}$ in animals expressing the hM3Dq in dmPFC was without effect.

Finally, the specific dmPFC cell types responsible for this effect remain unclear. Both hSYN-hM3Dq and CaMKII $\alpha$-hM3Dq DREADDs were effective in restoring fear prediction error and fear learning, and there was no difference between the two groups in terms of the strength of this restoration. This shows that pharmacogenetic excitation of dmPFC glutamatergic neurons is sufficient for restoration of fear prediction error and fear learning. However, injection of $\mathrm{CNO}$ induced c-Fos in both dmPFC hM3Dq-expressing and non-hM3Dq-expressing cells, even using the hSyn promoter (for similar findings, see Sasaki et al., 2011; Garner et al., 2012; Zhan et al., 2013), showing that the effects of pharmacogenetic excitation were not restricted to dmPFC hM3Dq-expressing cells. Whether these nonhM3Dq cells received direct synaptic input from hM3Dq cells or whether this reflects indirect network activation is unclear.

\section{dmPFC and fear prediction errors}

Consistent with computational (Rushworth and Behrens, 2008; Alexander and Brown, 2011), electrophysiological (Matsumoto et al., 2007), and neuroimaging (Corlett et al., 2004, 2006; Dunsmoor et al., 2008; Eippert et al., 2012) findings, our data causally implicate dmPFC in prediction errors showing that increases in dmPFC neuronal activity are sufficient to restore errors in fear predictions and to promote fear learning. Past attempts to establish a causal relation between dmPFC and fear learning have yielded inconsistent results (Morgan and Ledoux, 1995; Johansen and Fields, 2004; Corcoran and Quirk, 2007; Furlong et al., 2010; Sharpe and Killcross, 2014). At least one reason for this is that these past studies either have not behaviorally isolated the actions of prediction error or dmPFC has been manipulated by nonselective techniques (e.g., lesions), making inferences about dmPFC function difficult.
$\mathrm{dmPFC}$ has extensive projections to various amygdala nuclei, including basolateral amygdala (BLA; McDonald, 1998; Mátyás et al., 2014), and is well positioned to provide a prediction error input instructing amygdala-dependent learning and synaptic plasticity. BLA neurons are sensitive to fear prediction error in vitro (Bauer et al., 2001) and in vivo (Paton et al., 2006; Belova et al., 2007, 2008; Johansen et al., 2010), and similar findings have been reported in human neuroimaging studies (Dunsmoor et al., 2008). Of greatest relevance, there is bidirectional fear prediction error signaling between primate dorsal ACC (dACC) and amygdala (Klavir et al., 2013), and prediction error-related BOLD signals in human dACC precede changes in the amygdala BOLD signal during learning (Eippert et al., 2012). Thus, it is likely that dmPFC-BLA interactions contribute to the restoration of prediction error and fear learning observed here.

It is well documented that MPFC is critical for regulating the expression of fear. dmPFC, especially prelimbic PFC, is important for promoting fear expression (Corcoran and Quirk, 2007; Santini et al., 2008; Burgos-Robles et al., 2009; Senn et al., 2014) and ventromedial PFC (vmPFC), especially infralimbic PFC, is important for preventing fear expression such as during extinction or safety (Milad and Quirk, 2002; Phelps et al., 2004; Kalisch et al., 2006; Quirk et al., 2006; Vidal-Gonzalez et al., 2006; Schiller et al., 2008; Senn et al., 2014). However, the role for PFC is not limited just to regulating the expression of fear; it also includes regulating how much fear is actually learned.

Under normal conditions, increases in dmPFC activity signal unexpected aversive events to support fear learning, but as the aversive outcome becomes expected, dmPFC activity diminishes, as does further fear learning (Dunsmoor et al., 2008; Eippert et al., 2012). It is possible that just as reduced fear inhibition in clinical anxiety has been linked to underactivation of vmPFC (Milad et al., 2009), so too may the overprediction of fear that characterizes clinical anxiety (Cox and Swinson, 1994; Rachman, 1994; Schmidt et al., 1994; van Hout and Emmelkamp, 1994) be linked to overactivation of dmPFC. Moreover, human fMRI studies have linked excessive dlPFC-based prediction error signals with the severity of delusional beliefs, and have invoked these aberrations as a causal mechanism in the formation of delusions (Corlett et al., 2007). The present pharmacogenetic approach could prove useful to model the cellular and circuit-level consequences of such an excessive prediction error.

\section{Conclusions}

We combined pharmacogenetic techniques with a behavioral approach isolating the actions of fear prediction errors to show a causal relation between the activity of rat dmPFC neurons and fear prediction error. The injection of CNO in dmPFC hM3Dqexpressing animals restored prediction error and fear learning under conditions where these were absent, but was otherwise without effect on fear learning, memory consolidation, or retrieval. There was no evidence here that $\mathrm{dmPFC}$ hM3Dq DREADD expression or CNO injection alone altered fear learning or fear behaviors. We suggest that, in addition to its well established role in fear responding, $\mathrm{dmPFC}$ has an important role in fear prediction errors and that this dual role enables a signature of adaptive behavior: using the past to predict the future, and learning as well as responding appropriately.

\section{References}

Alexander GM, Rogan SC, Abbas AI, Armbruster BN, Pei Y, Allen JA, Nonneman RJ, Hartmann J, Moy SS, Nicolelis MA, McNamara JO, Roth BL 
(2009) Remote control of neuronal activity in transgenic mice expressing evolved G-protein coupled receptors. Neuron 63:27-39. CrossRef Medline

Alexander WH, Brown JW (2011) Medial prefrontal cortex as an actionoutcome predictor. Nat Neurosci 14:1338-1344. CrossRef Medline

Annau Z, Kamin LJ (1961) The conditioned emotional response as a function of the intensity of the US. J Comp Physiol Psychol 54:428-432. CrossRef Medline

Armbruster BN, Li X, Pausch MH, Herlitze S, Roth BL (2007) Evolving the lock to fit the key to create a familyof $\mathrm{G}$ protein-coupled receptors potentlyactivated by an inert ligand. Proc Natl Acad Sci U S A 104:5163-5168. CrossRef Medline

Bauer EP, LeDoux JE, Nader K (2001) Fear conditioning and LTP in the lateral amygdala are sensitive to the same stimulus contingencies. Nat Neurosci 4:687-688. CrossRef Medline

Belova MA, Paton JJ, Morrison SE, Salzman CD (2007) Expectation modulates neural responses to pleasant and aversive stimuli in primate amygdala. Neuron 55:970-984. CrossRef Medline

Belova MA, Paton JJ, Salzman CD (2008) Moment-to-moment tracking of state value in the amygdala. J Neurosci 28:10023-10030. CrossRef Medline

Bevins RA, Ayres JJB (1991) Two issues in Pavlovian fear conditioning: selective fear of bright vs. dark, and CS determinants of CR form. Behav Proc 24:211-218. CrossRef Medline

Burgos-Robles A, Vidal-Gonzalez I, Quirk GJ (2009) Sustained conditioned responses in prelimbic prefrontal neurons are correlated with fear expression and extinction failure. J Neurosci 29:8474-8482. CrossRef Medline

Corcoran KA, Quirk GJ (2007) Activity in prelimbic cortex is necessary for the expression of learned, but not innate, fears. J Neurosci 27:840-844. CrossRef Medline

Corlett PR, Aitken MR, Dickinson A, Shanks DR, Honey GD, Honey RA, Robbins TW, Bullmore ET, Fletcher PC (2004) Prediction error during retrospective revaluation of causal associations in humans: fMRI evidence in favor of an associative model of learning. Neuron 44:877888. Medline

Corlett PR, Honey GD, Aitken MR, Dickinson A, Shanks DR, Absalom AR, Lee M, Pomarol-Clotet E, Murray GK, McKenna PJ, Robbins TW, Bullmore ET, Fletcher PC (2006) Frontal responses during learning predict vulnerability to the psychotogenic effects of ketamine: linking cognition, brain activity, and psychosis. Arch Gen Psychiatr 63:611-621. CrossRef Medline

Corlett PR, Murray GK, Honey GD, Aitken MR, Shanks DR, Robbins TW, Bullmore ET, Dickinson A, Fletcher PC (2007) Disrupted predictionerror signal in psychosis: evidence for an associative account of delusions. Brain 130:2387-2400. CrossRef Medline

Cox BJ, Swinson RP (1994) Overprediction of fear in panic disorder with agoraphobia. Behav Res Ther 32:735-739. CrossRef Medline

Dickinson A (1980) Contemporary animal learning theory. Cambridge, UK: Cambridge UP.

Dunsmoor J, Schmajuk N (2009) Interpreting patterns of brain activation in human fear conditioning with an attentional-associative learning model. Behav Neurosci 123:851-855. CrossRef Medline

Dunsmoor JE, Bandettini PA, Knight DC (2008) Neural correlates of unconditioned response diminution during Pavlovian conditioning. Neuroimage 40:811-817. CrossRef Medline

Eippert F, Gamer M, Büchel C (2012) Neurobiological mechanisms underlying the blocking effect in aversive learning. J Neurosci 32:13164-13176. CrossRef Medline

Ferguson SM, Phillips PE, Roth BL, Wess J, Neumaier JF (2013) Directpathway striatal neurons regulate the retention of decision-making strategies. J Neurosci 33:11668-11676. CrossRef Medline

Furlong TM, Cole S, Hamlin AS, McNally GP (2010) The role of prefrontal cortex in predictive fear learning. Behav Neurosci 124:574-586. CrossRef Medline

Garner AR, Rowland DC, Hwang SY, Baumgaertel K, Roth BL, Kentros C, Mayford M (2012) Generation of a synthetic memory trace. Science 335: 1513-1516. CrossRef Medline

Harris RJ (2004) ANOVA: an analysis of variance primer. Itasca, IL: F.E. Peacock.

Johansen JP, Fields HL (2004) Glutamatergic activation of anterior cingu- late cortex produces an aversive teaching signal. Nat Neurosci 7:398-403. CrossRef Medline

Johansen JP, Tarpley JW, LeDoux JE, Blair HT (2010) Neural substrates for expectation-modulated fear learning in the amygdala and periaqueductal gray. Nat Neurosci 13:979-986. CrossRef Medline

Kalisch R, Korenfeld E, Stephan KE, Weiskopf N, Seymour B, Dolan RJ (2006) Context-dependent human extinction memory is mediated by a ventromedial prefrontal and hippocampal network. J Neurosci 26:95039511. CrossRef Medline

Kamin LJ (1968) Attention-like processes in classical conditioning. In: Miami symposium on the prediction of behavior: aversive stimulation (Campbell BJ, Church RM, eds), pp 9-33. Coral Gables, FL: University of Miami.

Klavir O, Genud-Gabai R, Paz R (2013) Functional connectivity between amygdala and cingulate cortex for adaptive aversive learning. Neuron 80:1290-1300. CrossRef Medline

Mackintosh NJ (1983) Conditioning and associative learning. Oxford: Oxford UP.

Maren S, Quirk GJ (2004) Neuronal signalling of fear memory. Nat Rev Neurosci 5:844-852. CrossRef Medline

Matsumoto M, Matsumoto K, Abe H, Tanaka K (2007) Medial prefrontal cell activity signaling prediction errors of action values. Nat Neurosci 10:647-656. CrossRef Medline

Mátyás F, Lee J, Shin HS, Acsády L (2014) The fear circuit of the mouse forebrain: connections between the mediodorsal thalamus, frontal cortices and basolateral amygdala. Eur J Neurosci 39:1810-1823. CrossRef Medline

McDonald AJ (1998) Cortical pathways to the mammalian amygdala. Prog Neurobiol 55:257-332. CrossRef Medline

McNally GP, Westbrook RF (2006) Predicting danger: the nature, consequences, and neural mechanisms of predictive fear learning. Learn Mem 13:245-253. CrossRef Medline

McNally GP, Johansen JP, Blair HT (2011) Placing prediction into the fear circuit. Trends Neurosci 34:283-292. CrossRef Medline

Milad MR, Quirk GJ (2002) Neurons in the medial prefrontal cortex signal memory for fear extinction. Nature 420:70-74. CrossRef Medline

Milad MR, Pitman RK, Ellis CB, Gold AL, Shin LM, Lasko NB, Zeidan MA, Handwerger K, Orr SP, Rauch SL (2009) Neurobiological basis of failure to recall extinction memory in posttraumatic stress disorder. Biol Psychiatry 66:1075-1082. CrossRef Medline

Morgan MA, LeDoux E (1995) Differential contribution of dorsal and ventral medial prefrontal cortex to the acquisition and extinction of conditioned fear in rats. Behav Neurosci 109:681-688. CrossRef Medline

Paton JJ, Belova MA, Morrison SE, Salzman CD (2006) The primate amygdala represents the positive and negative value of visual stimuli during learning. Nature 439:865-870. CrossRef Medline

Paxinos G, Watson C (2007) The rat brain in stereotaxic coordinates, 7th edition. Amsterdam: Elsevier.

Phelps EA, Delgado MR, Nearing KI, LeDoux JE (2004) Extinction learning in humans: role of the amygdala and vmPFC. Neuron 43:897-905. CrossRef Medline

Quirk GJ, Garcia R, González-Lima F (2006) Prefrontal mechanisms in extinction of conditioned fear. Biol Psychiatry 60:337-343. CrossRef Medline

Rachman S (1994) The overprediction of fear: a review. Behav Res Ther 32:683-690. CrossRef Medline

Rescorla RA, Wagner AR (1972) A theory of Pavlovian conditioning: variations in the effectiveness of reinforcement and nonreinforcement. In: Classical conditioning II: current research and theory (Black AH, Prokasy WF, eds), pp 64-99. New York: Appleton-Century Crofts.

Rogan SC, Roth BL (2011) Remote control of neuronal signaling. Pharmacol Rev 63:291-315. CrossRef Medline

Rushworth MF, Behrens TE (2008) Choice, uncertainty and value in prefrontal and cingulate cortex. Nat Neurosci 11:389-397. CrossRef Medline

Santini E, Quirk GJ, Porter JT (2008) Fear conditioning and extinction differentially modify the intrinsic excitability of infralimbic neurons. J Neurosci 28:4028-4036. CrossRef Medline

Sasaki K, Suzuki M, Mieda M, Tsujino N, Roth B, Sakurai T (2011) Pharmacogenetic modulation of orexin neurons alters sleep/wakefulness states in mice. PLoS One 6:e20360. CrossRef Medline 
Schiller D, Levy I, Niv Y, LeDoux JE, Phelps EA (2008) From fear to safety and back: reversal of fear in the human brain. J Neurosci 28:11517-11525. CrossRef Medline

Schmidt NB, Jacquin K, Telch MJ (1994) The overprediction of fear and panic in panic disorder. Behav Res Ther 32:701-707. CrossRef Medline

Senn V, Wolff SB, Herry C, Grenier F, Ehrlich I, Gründemann J, Fadok JP, Müller C, Letzkus JJ, Lüthi A (2014) Long-range connectivity defines behavioral specificity of amygdala neurons. Neuron 81:428-437. CrossRef Medline

Sharpe MJ, Killcross S (2014) The prelimbic cortex contributes to the downregulation of attention toward redundant cues. Cereb Cortex 24:10661074. CrossRef Medline
Sutton RS (1988) Learning to predict by the methods of temporal differences. Mach Learn 3:9-44. CrossRef

van Hout WJ, Emmelkamp PM (1994) Overprediction of fear in panic disorder patients with agoraphobia: does the (mis)match model generalize to exposure in vivo therapy? Behav Res Ther 32:723-734. CrossRef Medline

Vidal-Gonzalez I, Vidal-Gonzalez B, Rauch SL, Quirk GJ (2006) Microstimulation reveals opposing influences of prelimbic and infralimbic cortex on the expression of conditioned fear. Learn Mem 13:728-733. Medline

Zhan C, Zhou J, Feng Q, Zhang JE, Lin S, Bao J, Wu P, Luo M (2013) Acute and long-term suppression of feeding behavior by POMC neurons in the brainstem and hypothalamus, respectively. J Neurosci 33:3624-3632. CrossRef Medline 